\title{
Modelado por redes neuronales artificiales de los indicadores de desempeño de operación en instalaciones de gasificación termoquímica downdraft
}

Modeling by artificial neural networks of the performance indicators of the operation of a downdraft thermochemical gasifier

\author{
Eduardo Roberto Gutiérrez Gualotuña ${ }^{1}$, Juan Carlos Almeida Mera ${ }^{1}$ y \\ José Arzola Ruiz ${ }^{2}$
}

\section{RESUMEN}

En el trabajo se presentan resultados investigativos obtenidos por los autores en el modelado de indicadores de desempeño de la operación de instalaciones de gasificación de la biomasa con ayuda de redes neuronales artificiales, a partir de la clasificación de la información derivada del análisis sistémico de su operación. Se realizó el estudio bibliográfico de los trabajos de investigaciones previos relacionados. A partir de un plan experimental $3^{\mathrm{N}}$ con tres réplicas se elaboran, utilizando las técnicas de las redes neuronales, cuatro modelos correspondientes a respectivos indicadores de desempeño, los que se comparan con los obtenidos por modelos de regresión no lineal. Como biomasa se utiliza el Oryza sativa.

Palabras Clave: Operación de procesos tecnológicos; análisis de sistemas; fuentes de energía renovable; redes neuronales artificiales.

\footnotetext{
1 Universidad de las Fuerzas Armadas del Ecuador-ESPE. Sangolquí, Ecuador.

2 Centro de Estudios de Matemáticas para las Ciencias Técnicas-CEMAT, Universidad Tecnológica de La Habana José Antonio Echeverría. La Habana, Cuba.

(C) Los autores. Este artículo es publicado por la Revista Aporte Santiaguino de la Universidad Nacional Santiago Antúnez de Mayolo. Este es un artículo de acceso abierto, distribuido bajo los términos de la Licencia Creative Commons Atribución-NoComercial-CompartirIgual 4.0 Internacional. (http://creativecommons.org/licenses/ by-nc-sa/4.0/), que permite el uso no comercial, distribución y reproducción en cualquier medio, siempre que la obra original sea debidamente citada.
} 


\begin{abstract}
The research results are presented by the authors in the modeling of performance indicators of the operation of biomass gasification installations using artificial neural networks, starting by the classification of the information derived from the systemic analysis of its operation. A bibliographical study of previous related researches was developed. A $3^{\mathrm{N}}$ experimental plan with 3 replicas is made in order to generate four models according to their own performance indicators using neural networks. The quality indicators of the neural networks are compared with those obtained by nonlineal regression models. As biomass the Oryza sativa is used.
\end{abstract}

Keywords: Operation of technological processes; systems analysis; renewable energy sources; artificial neural networks.

\title{
INTRODUCCIÓN
}

La gasificación es la conversión de un caudal sólido en un combustible gaseoso y/o líquido que puede ser quemado para liberar energía o utilizado para la producción de sustancias químicas valiosas (Shabbar y Janajreh, 2013), requiere de un agente gasificante para reordenar la estructura molecular de la biomasa y convertirla en un combustible útil (Bhattacharya et al., 2013; Shabbar y Janajreh, 2013). El gas de salida del proceso de gasificación tipo downdraft depende de la cantidad del carbono que se produce durante la pirólisis y del agente gasificante utilizado en el proceso, el que depende de la temperatura de pirólisis, la que determina el rompimiento de la molécula de la biomasa gracias a la reacción de reducción que se produce por la cantidad de calor existente en el reactor y de la presión en el mismo. La calidad del gas que produce la instalación depende no solo del tipo de biomasa y del diseño del reactor, sino también de los parámetros de operación (Azzone, 2013; Sekhar y et al., 2013; Sharma, 2011).

Cuando el proceso transcurre para periodos pequeños de tiempo puede resultar conveniente la construcción de modelos matemáticos que predigan el comportamiento operacional de la instalación energética integralmente para todo el periodo de experimentación (Xie et al., 2012).

En el estudio bibliográfico realizado se refieren numerosos trabajos sobre diferentes tipos de modelos del proceso, algunos de ellos basados en el equilibrio termodinámico, los que se utilizan para predecir la composición del gas de síntesis al suponer una mezcla de reactivos en un tiempo infinito (Puig-Arnavat et al., 2013; Nguyen et al., 2013). El problema del empleo de este método es la introducción de errores, ya que no se toman en cuenta todas las reacciones existentes en el proceso (Bhattacharya et al. 2013; Pirc et al., 2013). En cambio, al emplear un modelo no estequiométrico se minimiza la energía libre de Gibbs (Azzone et al., 2013; Shabar et al., 2013) sin tomar en cuenta la 
presencia de las diferentes reacciones. Este tipo de modelos da preferencia a los parámetros termodinámicos sin referencia a los parámetros de operación de la instalación en sí, para un análisis de los componentes existentes en el gas de síntesis, por lo que los errores de apreciación del desempeño siguen siendo considerables.

Otro tipo modelos importante es el cinético. Al igual que el modelo anterior se enfoca en realizar un análisis del gas de síntesis producto del proceso y predecir su rendimiento en función de su composición en un volumen de control en un tiempo dado (Janajreh et al., 2013). En algunos trabajos estos modelos se validan con ayuda de resultados experimentales (Sreejith et al., 2013). Con ayuda de este tipo de modelos se predicen los perfiles tanto de composición del gas como del perfil de temperatura dentro del reactor en condiciones de operación dadas. Aquí se considera la cinética de las diferentes reacciones y también las condiciones de movimiento dela biomasa procesada dentro del reactor (DiBlasi, 2000; Puig-Arnavat et al., 2010; Tigabwa et al., 2012), es decir, su hidrodinámica, balances de masa y energía requeridas enfocadas hacia el conocimiento del rendimiento del gas. Al igual que el método de equilibrio termodinámico, este método se enfoca de manera muy especial a la caracterización del gas de síntesis y de sus componentes, dando especial cuidado a la mezcla y su rendimiento, siendo muy sensible a la reacción dada en dos fases (sólido-gas) dentro del reactor, por lo que se puede decir que estos modelos son más precisos que los anteriores y detallan el proceso de gasificación, aunque son computacionalmente muy intensivos (Akay y Jordan, 2011; Sekhar et al., 2013).

Los modelos basados en la dinámica de fluidos computacional (CFD) permiten realizar una simulación rápida y eficaz del flujo de fluidos y los procesos de transferencia de calor y masa dentro del reactor, con lo que se pueden calcular las fuerzas que actúan en el fluido y ayuda a comprender el impacto del gas en el rendimiento de la instalación de gasificación. Se basan en la solución de ecuaciones simultaneas obtenidas de los balances de energía, masa y momento en cualquier zona del reactor, para predecir y formular perfiles de temperatura. Estos modelos se han utilizado para caracterizar el comportamiento de varios tipos de gasificadores de biomasa al tomar en cuenta que la composición de la biomasa es compleja y depende de la materia prima, tiempo a la intemperie, ubicación geográfica y época del año (Baruah et al., 2014).

En los enfoques anteriores se destaca la falta de integralidad, pues cada uno de ellos describe el proceso considerando una parte de los complejos procesos que tienen lugar dentro del reactor, lo que conduce a errores que aportan los modelos de origen químico-físico del proceso de gasificación, por lo que sugiere la idea de la realización de experimentos que permitan elaborar modelos para la operación de los procesos, siendo los errores obtenidos dependientes de la representatividad del tipo de modelo. Entre ellos se encuentra el modelado por técnicas de regresión lineal realizado por Vladimir 
Kirsanovs y colaboradores (Kirsanovs et al. 2017). En este trabajo el autor realiza una investigación experimental del proceso de gasificación en una planta real de 400 kW con el fin de caracterizar indicadores de eficiencia de la operación. Se hallan cuatro modelos para definir el poder calórico del gas de síntesis, la capacidad del gasificador y la eficiencia en frio y en caliente del gas. No obstante, carece de un análisis sistémico previo que permita definir el conjunto de relaciones que se requiere modelar con el fin de operar racionalmente el proceso. En el trabajo de Tigabwa y colaboradores (Tigabwa et al., 2012), se da prioridad a la generación de hidrogeno como producto del proceso de gasificación y se hace una revisión de los modelos utilizados, entre ellos las redes neuronales artificiales (RNA). Las mismas que se usan para mejorar la precisión de predicción de la composición del gas de síntesis (Akay y Jordan, 2011). Sin embargo, el modelado por redes neuronales requiere de datos experimentales a partir de un plan experimental, los que no se reflejan en la publicación. De igual forma, Puig-Arnavat (Puig-Arnavat et al., 2013), publica un trabajo sobre modelado con RNAdel proceso de gasificación de biomasa en gasificadores tipo downdraft. Estos modelos se formularon para predecir la composición del gas de síntesis en términos de concentración de cuatro componentes principales: $\mathrm{CH}_{4}, \mathrm{CO}, \mathrm{CO}_{2} \mathrm{y} \mathrm{H}_{2} \mathrm{y}$, al igual que los anteriormente referenciados no se sustentan en estudio sistémico alguno orientado al diseño o a la operación. En otros muchos trabajos (Akay y Jordan, 2011; Puig et al., 2010) aparecen también otros modelos basados en RNA, los que se dedican al estudio de la gasificación de la biomasa y de la producción de carbón y su gasificación sin resultados útiles para la operación del proceso tecnológico.

En adelante, el modelado se realiza con ayuda de las RNA a partir del análisis sistémico dela operación de este tipo de instalaciones que permite definir el conjunto de relaciones requeridas para modelarla, para la gasificación de diferentes biomasas, siendo descritos los modelos obtenidos correspondientes a la biomasa Oryza sativa (cáscara de arroz) en una instalación experimental construida con el objetivo de generar los datos experimentales requeridos para identificar las relaciones que deben forman parte de los modelos de toma de decisiones de operación. Los modelos obtenidos por redes neuronales se comparan con los obtenidos por modelos no lineales de regresión. Una vez en funcionamiento el sistema, se hará posible obtener abundante información estadística como resultado del experimento pasivo resultante de su operación normal, lo que ha de permitir perfeccionar las relaciones que intervienen con mucha mayor precisión, como resultado del volumen de datos que se habría de acumular, lo que permitiría obtener modelos mucho más precisos.

\section{MATERIALES Y MÉTODOS}

La instalación experimental está constituida por un gasificador tipo downdraft con una capacidad de $10 \mathrm{~kW}$ el que se conecta a un motor de combustión interna de 0,8 
$\mathrm{kW}$ de potencia nominal como carga. Como resultado del análisis sistémico realizado en correspondencia con la metodología de análisis y síntesis de sistema de ingeniería expuesta en (Arzola-Ruiz, 2012), se obtiene la siguiente composición de variables para la tarea de operación de la instalación de gasificación downdraft.

Variables de coordinación: Potencia demandada (o deseada) $u^{d}$, humedad de la biomasaHu y poder calórico inferior requerido del gas generado $P C G^{\text {inf }}$,

Variables de decisión: Cantidad de biomasa del tipo j Cbio y caudal de aire de combustión Cau.

Indicadores de eficiencia: Eficiencia de la instalación Ef, temperatura de la zona pilórica Tzp, poder calórico del gas PCG y caudal másico de gas generado $M G$.

Como salidas del análisis externo se obtienen el modelo matemático conceptual de operación y las relaciones que deben ser identificadas. En calidad de estas últimas se tienen:

$$
\begin{aligned}
& E f=E f(H u, C b i o, \text { Cau }) \\
& T_{z p}=\operatorname{Tzp}(H u, C b i o, \text { Cau }) \\
& M G=M G(H u, C b i o, \text { Cau }) \\
& P C G=P C G(H u, C b i o, C a u)
\end{aligned}
$$

Con el fin de identificar estas relaciones, se concibió un plan experimental con una estructura $3^{\mathrm{N}}$ (alto, medio y bajo, por variables independientes) con tres réplicas, que permitan reflejar los errores casuales, incluyendo los de medición, para biomasas con bajo, medio y alto poder calórico. Para medio poder calórico fue seleccionado el Oryza sativa, objeto de identificación en el presente artículo. Se seleccionaron los niveles, para las variables independientes, de: $12 \%, 16 \%, 20 \%$ para $H u ; 1 \mathrm{~kg}, 3 \mathrm{~kg}, 5 \mathrm{~kg}$ para Cbio; 0,0001666 kg/s, 0,0002098 kg/s, 0,0002531 kg/s para Cau. Para la recopilación automatizada de la información experimental, se utilizó en la instalación una tarjeta de adquisición de datos myRIO y la interface maquina hombre con un programa desarrollado en LabView. La instrumentación utilizada consta de una alarma digital de detección de CO marca Kidde con una intervalo de medición de 30-999 PPM con error $\pm 30 \%$ de la magnitud medida en condiciones normales, un medidor de humedad MD914 con un intervalo de medición de 2 a 60\% una resolución de $0,5 \%$ un sensor de nivel de combustible controlado mediante una paleta para censar el nivel de biomasa, 8 termopares tipo $\mathrm{k}$, con intervalo de medición de 0 a $1250^{\circ} \mathrm{C}$ de temperatura con un límite de error $\pm 2,2{ }^{\circ} \mathrm{C}$ de chromel - alumel y un cable de compensación $\mathrm{KX}$, ubicadas de la siguiente forma: cuatro para las zonas de reducción, pirolisis, combustión y secado, una en la descarga de cenizas, y tres para censar las temperaturas del gas a la entrada 
del ciclón, a la entrada del intercambiador de calor y a la salida del intercambiador de calor, así como de dos manómetros de agua tipo U con intervalo de medición de 200 $\mathrm{mm}$ de $\mathrm{H}_{2} \mathrm{O}$, con error de medición de $\pm 1 \mathrm{~mm}$ de $\mathrm{H}_{2} \mathrm{O}$. Uno de ellos toma la diferencia de presión existente en el reactor y el otro en la placa orificio para medición del caudal másico de aire que ingresa en las diferentes aperturas de válvula. La disposición de toda la instrumentación se ilustra en la figura 1.

Los experimentos se realizaron por lotes en el siguiente orden: activación de la instalación, carga de la biomasa, apertura de la válvula de aire, captura del gas resultante y envío a laboratorio para la medición de composición y masa de los gases y cálculo de su poder calórico. La medición de temperatura se realizaba de forma permanente en los puntos indicados en la figura 1 y se promediaba la temperatura en la zona pilórica para todo el lote.

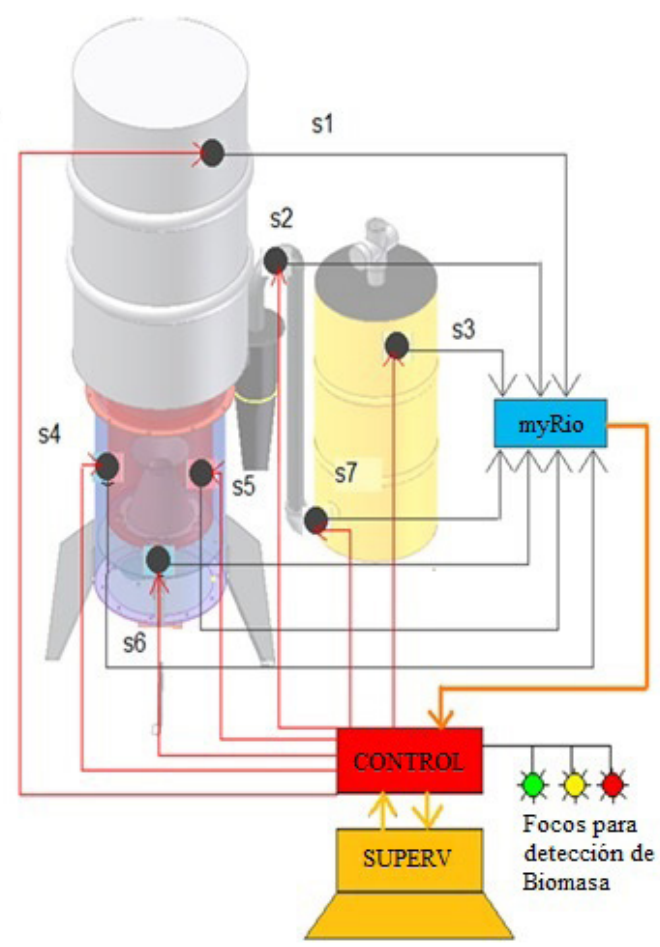

Figura 1. Puntos de control del gasificador tipo downdraft

Para crear las redes neuronales se utiliza una herramienta programada en $M A T L A B$ (Arbib et al., 2002), la que permite crear y manipular objetos en base a redes neuronales, con número variable de capas ocultas y de nodos por capa, función de activación para los nodos de las capas ocultas, y optimización de los valores de los pesos entre nodos y de los valores de las vías por capas. El software realiza, además, toda la gestión de información de entrada - salida de forma de poder variar operativamente la confi- 
guración de la red visualizado el comportamiento de los indicadores $R$ y error estándar óptimos de la configuración para los datos seleccionados para entrenar la red y para validarla.

\section{RESULTADOS}

Se elaboraron variantes de redes neuronales con la inclusión de todas las salidas en un mismo modelo, pero dada la muy baja correlación entre los indicadores de eficiencia y la poca cantidad de datos experimentales para el entrenamiento de la red, se evidenció la necesidad del procesamiento individual de los indicadores de desempeño de la operación del proceso.

Para el entrenamiento de las redes los datos se seleccionaron aleatoriamente en cada corrida. Se adoptó el 30\% para la validación y el 70\% para el entrenamiento de la red neuronal. Como resultado, se estructuraron redes con 1 y 2 capas ocultas. En todos los casos la estructura de dos capas ocultas, con funciones de trasferencia no lineales, adicionalmente a las capas de entrada y salidas de datos resultó la más efectiva. Para las dos capas ocultas la cantidad de nodos requeridos y de las funciones de transferencia se establecieron mediante prueba y error, en correspondencia con el plan experimental señalado, para modelar las variables de salida $y=\left(E f, T_{z p}, M G\right.$ y $\left.P C G\right)$, utilizando como objetivo el máximo valor de $R$. En la figura 2 se muestra gráficamente la estructura de la red finalmente obtenida.

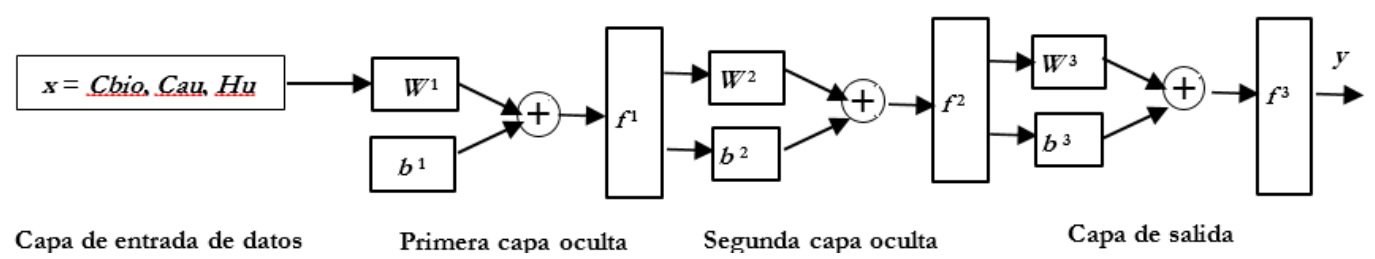

Figura 2. Estructura de la red neuronal encontrada para todas y cada una de las salidas

En los resultados se identifican, de tal forma, modelos con la estructura general dada por:

$$
y=f^{3}\left(W^{3} f^{2}\left(W^{2} f^{1}\left(W^{1} x+b^{1}\right)+b^{2}\right)+b^{3}\right),
$$

donde $y$ representa la variable de salida modelada, $x$ el vector de entrada, $W^{i}, f, b^{i}, n^{i}$ la matriz de coeficientes, función de transición, vector de vías y salidas de la capa $i$. 
Los mejores resultados se obtuvieron, para los cuatro modelos buscados, ocho nodos en la primera capa oculta y cuatro en la segunda oculta. En la tabla 1 se muestran los resultados intermedios obtenidos durante la búsqueda del número de nodos óptimo por capas durante la optimización de la red para el modelado de Ef.

Tabla 1. Búsqueda del número óptimo de nodos para redes ocultas en el modelado de Ef

\begin{tabular}{ccccc}
\hline No. de Modelo & Capa 2 & Capa 3 & R Entrenamiento & RValidación \\
\hline 1 & 10 & 6 & 0,7425 & 0,8596 \\
2 & 8 & 6 & 0,9124 & 0,7048 \\
3 & 6 & 6 & 0,8125 & 0,7746 \\
4 & 10 & 4 & 0,8025 & 0,8326 \\
$\mathbf{5}$ & $\mathbf{8}$ & $\mathbf{4}$ & $\mathbf{0 , 8 2 5 5}$ & $\mathbf{0 , 8 5 3 2}$ \\
6 & 6 & 4 & 0,7632 & 0,8034 \\
7 & 10 & 2 & 0,7401 & 0,8042 \\
8 & 8 & 2 & 0,7445 & 0,7736 \\
9 & 6 & 2 & 0,7426 & 0,7715 \\
\hline
\end{tabular}

La función de trasferencia más adecuada en las capas ocultas 1 y 2, para los cuatro modelos, resultó ser la función tangente sigmoidal. Esta función entrega una salida que se encuentra en el rango de $(-1,0$ a 1,0$)$ y es utilizado con frecuencia en redes del tipo estudiado.

En adelante, se muestran las matrices y vías óptimos obtenidos para el modelo de Ef:

$$
\begin{aligned}
& W_{1}=\left[\begin{array}{ccc}
-0,266 & 2,965 & 7,127 \\
3,409 & 1,237 & 1,127 \\
-2,063 & 1,950 & -3,255 \\
-3,286 & 2,660 & -2,736 \\
5,255 & -6,088 & -0,179 \\
-5,169 & 0,513 & 5,137 \\
0,082 & 0,267 & 1,969 \\
0,947 & 6,028 & -3,306
\end{array}\right] ; \quad b_{1}=\left[\begin{array}{c}
-0,437 \\
-1,265 \\
-4,000 \\
0,590 \\
-3,469 \\
-3,674 \\
-3,691 \\
0,428
\end{array}\right] \\
& W_{2}=\left[\begin{array}{cccccccc}
-0,502 & 1,153 & 2,628 & 1,4421 & 3,075 & -1,273 & 0,227 & -1,596 \\
-1,633 & -1,375 & 3,088 & -1,521 & -0,853 & -0,404 & 0,162 & -1,333 \\
-1,463 & 1,824 & -0,553 & 1,309 & 0,038 & 0,194 & 6,532 & 2,905 \\
-1,021 & 0,104 & 1,523 & 1,13 & -1,021 & -0,966 & -0,925 & -0,301
\end{array}\right] ; b_{2}=\left[\begin{array}{c}
0,028 \\
0,975 \\
-1,583 \\
-1,588
\end{array}\right] \\
& W_{3}=\left[\begin{array}{llll}
-0,677 & -0,393 & 0,550 & 1,319
\end{array}\right] ; b_{3}=[0,701]
\end{aligned}
$$


Se obtuvieron el coeficiente de regresión y el error estándar (desviación típica) calculados para los datos de validación de la red, respectivamente, $R=85,32 ; S=1,53$

Estos resultados se comparan con la estimación de Ef, con ayuda de un modelo de regresión no lineal de ajuste, obtenida a partir de los mismos datos experimentales:

$E f=-61,74+72,60 \mathrm{Cau}^{0,2} \mathrm{Hu}^{-0.21}-107,17 \mathrm{Cbio}^{0,08}+2,18 \mathrm{Hu}+236,99 \mathrm{Hu}^{-0,21} \mathrm{Cbio}^{0,08}$

Con los indicadores $R=77,5 ; S=2,03$

Matrices y vías óptimas obtenidas para el modelo de Trp:

$$
\begin{aligned}
& W_{1}=\left[\begin{array}{ccc}
1,694 & 1,006 & 0,9164 \\
-1,381 & 2,377 & -0,691 \\
1,381 & 1,631 & -2,394 \\
0,628 & -0,191 & -3,283 \\
-2,590 & -1,004 & -0,785 \\
-0,576 & 1,148 & 3,380 \\
0,057 & -0,935 & 2,490 \\
-0,626 & 2,938 & 0,750
\end{array}\right] ; b_{1}=\left[\begin{array}{c}
-3,372 \\
1,447 \\
1,976 \\
-0,93 \\
-0,272 \\
-0,428 \\
2,431 \\
-2,067
\end{array}\right] \\
& W_{2}=\left[\begin{array}{cccccccc}
0,116 & -0,1 & 0,558 & -0,670 & 0,004 & -1,461 & 0,312 & 1,351 \\
-0,45 & 2,074 & 0,140 & 0,842 & 0,866 & 0,152 & -0,503 & 0,731 \\
0,691 & -1,032 & -0,031 & 1,029 & 0,294 & -0,651 & 0,199 & -1,470 \\
0,813 & 0,099 & -0,651 & 0,823 & -0,365 & -0,102 & 0,77 & 0,409
\end{array}\right] ; b_{2}=\left[\begin{array}{c}
-1,555 \\
0,844 \\
0,561 \\
0,672
\end{array}\right] \\
& W_{3}=\left[\begin{array}{llll}
1,445 & 0,519 & 0,168 & -0,721
\end{array}\right] ; b_{3}=[0,500]
\end{aligned}
$$

Se obtuvieron el coeficiente de regresión y el error estándar calculados para los datos de validación de la red, respectivamente, $R=89,85 ; S=4,32$

Estos resultados se comparan con la estimación de Tzpcon ayuda de un modelo de regresión no lineal de ajuste, obtenida a partir de los mismos datos experimentales: Tzp $=538202-543003 \mathrm{Cau}^{0,003}+2431,73 \mathrm{Cau}^{0,003} \mathrm{Cbio}^{0,061}+458863 \mathrm{Cau}^{0,003} \mathrm{Hu}^{-0,284}$ $-1955,51 \mathrm{Cau}^{0,003} \mathrm{Hu}^{-0,284} \mathrm{Cbio}^{0,061}-454772 \mathrm{Hu}^{-0,284}$

Con los indicadores $R=83,40 ; S=4,34$.

Matrices y vías óptimas obtenidas para el modelo de $M G$ :

$$
W_{1}=\left[\begin{array}{ccc}
-0,938 & 2,980 & 0,769 \\
2,759 & -1,320 & -0,193 \\
2,499 & 1,705 & 0,178 \\
1,958 & 0,117 & -1,541 \\
2,226 & -0,223 & 2,133 \\
0,585 & -0,266 & 1,749 \\
-2,397 & 1,167 & -1,610 \\
1,517 & -0,060 & 2,509
\end{array}\right] ; b_{1}=\left[\begin{array}{c}
2,3291 \\
-1,9335 \\
0,0018 \\
-0,7852 \\
-1,4797 \\
1,0604 \\
-1,4422 \\
2,642
\end{array}\right]
$$




$$
\begin{aligned}
& W_{2}=\left[\begin{array}{cccccccc}
0,412 & -0,581 & -0,201 & 1,400 & -0,680 & -0,798 & -0,659 & -1,300 \\
0,586 & -0,743 & 0,188 & -1,053 & -0,021 & -0,667 & 1,400 & 1,344 \\
-0,096 & -0,212 & -1,243 & -0,774 & -0,941 & -0,315 & 0,395 & -0,807 \\
0,413 & -0,234 & 0,029 & -1,029 & -1,18 & -0,875 & 0,818 & 0,132
\end{array}\right] ; b_{2}=\left[\begin{array}{c}
-1,555 \\
0,844 \\
0,561 \\
0,672
\end{array}\right] \\
& W_{3}=\left[\begin{array}{lllll}
-2,148 & 0,978 & 1,014 & -0,512
\end{array}\right] ; b_{3}=[0,039]
\end{aligned}
$$

Se obtuvieron el coeficiente de regresión y el error estándar calculados para los datos de validación de la red, respectivamente, $R=96,6 ; S=0,15$

Estos resultados se comparan con la estimación de $M G$ con ayuda de un modelo de regresión no lineal de ajuste, obtenida a partir de los mismos datos experimentales:

$M G=5,07-1454,41 \mathrm{Cau}^{3,59} \mathrm{Cbio}^{0,01}+1366,2 \mathrm{Cau}^{3,59} \mathrm{Hu}^{0,09} \mathrm{Cbio}^{0,01}-3,88 \mathrm{Hu}^{0,09} \mathrm{Cbio}^{0,01}$

Con los indicadores $R=93,02 ; S=0,18$

Matrices y vías óptimos obtenidos para el modelo de PCG:

$$
\begin{aligned}
& W_{1}=\left[\begin{array}{ccc}
-1,620 & 1,622 & 1,394 \\
-0,436 & 2,752 & 0,306 \\
1,591 & 2,070 & 0,281 \\
2,044 & -0,237 & -1,977 \\
1,528 & -0,315 & 1,986 \\
0,235 & -2,681 & -1,010 \\
2,114 & 1,490 & -1,903 \\
-0,568 & 1,701 & 1,967
\end{array}\right] ; b_{1}=\left[\begin{array}{c}
2,923 \\
2,094 \\
-1,528 \\
-0,722 \\
0,522 \\
-0,870 \\
1,489 \\
-2,950
\end{array}\right] \\
& W_{2}=\left[\begin{array}{cccccccc}
0,563 & 0,527 & 0,270 & -0,387 & 0,968 & 0,327 & 0,890 & -0,121 \\
0,774 & -0,915 & -0,538 & -0,187 & -0,681 & 0,949 & -0,170 & -0,461 \\
-0,349 & -0,016 & 1,310 & 0,844 & -0,893 & 0,431 & -0,173 & -0,604 \\
0,017 & -0,651 & 0,643 & 0,993 & 0,114 & -1,089 & -1,104 & -0,895
\end{array}\right] ; b_{2}=\left[\begin{array}{c}
-1,870 \\
-0,694 \\
-0,563 \\
1,583
\end{array}\right] \\
& W_{3}=\left[\begin{array}{llll}
-0,793 & -0,619 & 0,533 & -0,942
\end{array}\right] ; b_{3}=[0,643]
\end{aligned}
$$

Se obtuvieron el coeficiente de regresión y el error estándar calculados para los datos de validación de la red, respectivamente, $R=96,0 ; S=0,65$

Estos resultados se comparan con la estimación de PCG con ayuda de un modelo de regresión no lineal de ajuste, obtenida a partir de los mismos datos experimentales:

Para la estimación del poder calórico del gas (PCG), se puede observar en la ecuación 14: 
$P C G=-103,42+71,42 \mathrm{Cau}^{0,33} \mathrm{Hu}^{-0,22} \mathrm{Cbio}^{0,08}+45,18 \mathrm{Cbio}^{0,08}+0,84 \mathrm{Hu}+212,1 \mathrm{Hu}^{-0,22}-139,06 \mathrm{Hu}^{-0,22} \mathrm{Cbio}^{0,08}(9)$

Con los indicadores $R=84,8 ; S=0,76$

\section{DISCUSIÓN}

Los resultados obtenidos muestran la posibilidad de utilización de las RNA en la identificación de los indicadores de operación de las instalaciones de gasificación tipo downdraft. Las RNA obtenidas, para la identificación de todas y cada una de las relaciones requeridas para la operación del proceso de gasificación en la instalación experimental, tienen la misma estructura, tanto por la cantidad de nodos por capas como por las funciones de transferencia de los nodos por capas. No se logra en una misma red identificar todos los indicadores requeridos dada la ausencia de suficiente correlación entre ellos y la pequeña cantidad de datos de partida (81). A pesar de esto se logran indicadores estadísticos con suficiente precisión para la implementación de estas redes como un modelo inicial de operación, el que, en condiciones de instalaciones en explotación, debe ser rectificado periódicamente a partir del experimento pasivo derivado de ella, pues mientras mayor sea la cantidad de datos mayor será la calidad de los modelos obtenidos.

\section{CONCLUSIONES}

El trabajo realizado permite extraer las siguientes conclusiones principales:

La composición de modelos a ser identificados depende del adecuado análisis del sistema estudiado. En el caso concreto de la operación de las instalaciones de gasificación tipo downdraftse requieren identificarla eficiencia de la instalación (Ef), temperatura de la zona pilórica (Tحp), poder calórico del gas (PCG), caudal másico del gas (MG)como función de la humedad $(H u)$, cantidad de biomasa (Cbio) ycaudal de aire de combustión (Cau).

Los modelos obtenidos para la biomasa Oryza sativa brindan resultados mejores que los logrados por los autores utilizando modelos de regresión a partir de monomiales linealizados, lo que no significa que no puedan ser mejorados con ayuda de otros modelos no lineales.

Las redes neuronales elaboradas para una instalación con vistas a su operación pueden ser utilizadas tan solo como modelos iniciales. Los definitivos deben ser rectificados periódicamente a partir del experimento pasivo resultante de su operación normal, con el fin de adaptarlos para cada relación que forma parte del modelo general de operación. 
En ausencia de modelos alternativos que satisfagan las necesidades del análisis sistémico se requiere realizar planes experimentales con suficiente número de ensayos para lograr su identificación mediante los métodos de procesamiento de datos de elevada efectividad, entre los que se encuentran las redes neuronales artificiales.

La aplicación de estas redes al caso concreto de la operación delgasificador tipo downdraft desarrollado en la investigación experimental, para la biomasa Oryza Sativa, se obtienen redes individuales por cada indicador de desempeño de la operación con 2 capas ocultas, 8 nodos en la primera y cuatro en la segunda y funciones de transferencias tipo tangencial sigmoidal y valores de pesos y vías por capas propios de cada indicador concreto. Se puede considerar que estas redes sean de utilidad también en la identificación de otras instalaciones de gasificación tipo downdraft, como punto de partida de la construcción de modelos de operación del proceso de gasificación.

\section{REFERENCIAS BIBLIOGRÁFICAS}

Akay, Galip y Jordan, Andrea. 2011. «Gasification of Fuel Cane Bagasse in a Downdraft Gasifier: Influence of Lignocellulosic Composition and Fuel Particle Size on Syngas Composition and Yield».Energy Fuels, 25, 2274-2283. <doi: 10.1021/ef101494w>

Arzola-Ruiz, José, 2012. Sistemas de Ingeniería. La Habana: Editorial Félix Valera.

Arbib, Michael. 2002. The Handbook of Brain Theory and Neural Networks, second edition. THE MIT PRESS, Cambridge, Massachusetts London, England

Azzone, Emanuele; Morini, Mirko y Michele, Pinelli. 2013. «Development of an equilibrium model for the simulation of thermochemical gasification and application to agricultural residues». Renewable Energy, Vol. 46, pp. 248-54. <doi: 10.1016/j.renene.2012.03.017>

Baruah, Diapal y Baruah, D.C. 2014. «Modeling of biomass gasification». Renewable and Sustainable Energy Reviews39, 806-815. <doi: 10.1016/j.rser.2014.07.129>

Bhattacharya, Atmadeep; Bhattacharya, Abhishek; Datta, Amitava. 2012. «Modeling of hydrogen production process from biomass using oxygen blown gasification». Int Hydrogen Energy, Vol. 37: pp.18782-90. < doi: 10.1016/j.ijhydene.2012.09.131>

DiBlasi, C. 2000. «Dynamic behaviour of stratified downdraft gasifiers». Chemical Engineering Sciemces, Vol. 55: pp. 2931-2944. <doi:10.1016/S00092509(99)00562-X> 
Janajreh, I.; Al Shrah, M. 2013. «Numerical and experimental investigation of downdraft gasification of woodchips». Energy Conversion Managment, Vol. 65, pp.783-792. < doi:10.1016/j.enconman.2012.03.0091>

Kirsanovs, Vladimirs et al. 2017. «Experimental investigation of downdraft gasifier at various conditions» Energy Procedia, Vol. 128, pp. 332-338, doi.org/10.1016/j. egypro.2017.08.321.

Nguyen, Thanh; et al. 2013. «Three stage steady state model for biomass gasification in a dual circulating fluidized bed». Energy Conversion and Management, Vol.54 (1), 100-12. < doi:10.1016/j.enconman.2011.09.019>

Pirc, A; Sekavčnik, M. y Mori, M. 2013. «Universal model of a biomass gasifier for different syngas compositions». Journal of Mechanical Engineering, 58(2012)5, 291-299

Puig-Arnavat, María; Bruno, Juan Carlos y Coronas, Alberto. 2010. «Review and analysis of biomass gasification models». Renewable and Sustainable Energy Reviews. Vol. 14, 2010, pp. 2841-2851. <doi: 10.1016/j.biombioe.2012.12.012>

Puig-Arnavat, Maria; Hernández, Alfredo; Bruno, Joan Charles y Coronas, Alberto. 2013. «Artificial neural network models for biomass gasification in fluidized bed gasifiers». Biomass Bioenergy, Vol. 49, pp. 279-289. <doi: 10.1016/j. biombioe.2012.12.012>

Shabbar, Syed y Janajreh, Isam. 2013. «Thermodynamic equilibrium analysis of coal gasification using Gibbs energy minimization method». Energy Conversion and Management, Vol. 65, pp.755-63. <doi:10.1016/j.enconman.2012.02.032>

Sharma, Avdhesh. 2011. «Experimental investigations on a 20 kW, solid biomass gasification system». Biomass Bioenergy, Vol. 35 pp. 421-428. < doi:10.1016/j. biombioe.2010.08.060>

Sreejith, C.; Muraleedharan, C. y Arun, P. 2013. «Performance prediction of fluidized bed gasification of biomass using experimental data-based simulation models». Biomass Convers Biorefinery, Vol. 33, pp.1-22. < doi: 10.1007/s13399-013-0083-5>

Sekhar, Niladri; Ghosh, Sudip y De, Sudipta. 2013. «Gasification of biomass in a fixed bed downdraft gasifier: a realistic model including tar»Bioresour Technol; Vol. 107, pp 505-511. <doi: 10.1016/j.biortech.2011.12.124> 
Tigabwa Y. et al. 2012. «Mathematical and computational approaches for design of biomass gasificationfor hydrogen production: A review» Renewable and Sustainable Energy Reviews 16 2304-2315. < doi:10.1016/j.rser.2012.01.035>

Xie, Jun; Zhong, Wenqi; Jin, Baosheng; Shao, Yingjuan; Liu, Hao. 2012.«Simulation on gasification of forestry residues in fluidized beds by EulerianLagrangian approach». BioresourTechnol, Vol. 121:pp36-46. $<$ doi: 10.1016/j. biortech.2012.06.080>

Recepción: 24/08/2018

Aceptación: 20/10/2018

\section{Correspondencia}

Eduardo Roberto Gutiérrez Gualotuña

ergutierrez@espe.edu.ec 